URL: http://www.una.ac.cr/salud CORREO: revistamhsalud@una.cr

Revista MHSalud® (ISSN: 1659-097X) Vol. 5. No. 1. Julio, 2008.

\title{
GENERACIÓN TRAS GENERACIÓN, SE RECOBRAN LOS JUEGOS TRADICIONALES
}

\author{
María Morera Castro, Máster en Recreación \\ Universidad Nacional y Universidad de Auburn \\ Costa Rica \\ mrmc2479@gmail.com
}

\begin{abstract}
RESUMEN
Actualmente, las comunidades, barrios y ambientes se encuentran inmersos en una serie de situaciones y problemáticas que han propiciado el deterioro de valores sociales, culturales y espirituales, indispensables para la armonía con uno mismo, el prójimo y el entorno. Los estereotipos se han apoderado de las mentes y los entornos se han reducido a cuatro paredes, llenos de rejas y dispositivos de seguridad. La paz, la fraternidad, y alegría se van, disminuyen. Y es aquí donde la labor social, espiritual y profesional de especialistas en el ámbito recreativo toma fuerza para contribuir con el rescate y la reestructuración de la sociedad. Son los juegos y las rondas tradicionales instrumentos que facilitan las relaciones, contribuyen en el aprendizaje y son una prueba de demostración de habilidades. Forman parte inseparable de la vida de la persona ya que estos son una expresión social y cultural de la adaptación que ha protagonizado el ser humano en relación con su entorno (Maestro, 2005). Además no toma en cuenta la raza, edad, sexo o la condición social; son una fuente generadora de salud, una ayuda para mejorar las destrezas motoras, cognitivas y emocionales; un medio para fomentar la creatividad, despertar la imaginación, desarrollar el sentido del ritmo; su objetivo es el bienestar personal. Son un estímulo para liberar tensiones y energía acumulada, así como para salir de la rutina diaria y un puente para el aprendizaje de sí mismo, del hábitat, de valores, costumbres y tradiciones. A través de este documento las personas podrán encontrar respuesta a cómo se trasmiten los juegos tradicionales, qué los caracteriza, por qué son una herramienta importante en la sociedad actual, con qué y cómo se pueden elaborar y de qué manera se puede contribuir al rescate y conservación de los mismos.
\end{abstract}

PALABRAS CLAVES: juegos tradicionales, recreación, juego, costumbres, tradiciones

\section{RECOVERING TRADITIONAL GAMES GENERATION AFTER GENERATION}

ABSTRACT

Communities, neighborhoods, and other environments are currently immersed in a series of situations and problems that have favored the deterioration of social, cultural and spiritual values, which are essential for harmony with oneself, others, and the environment. Stereotypes have captured minds and settings have been reduced to indoor spaces, hemmed in by security bars and protective devices. Peace, fraternity and happiness are diminishing. It is at this point that the social, spiritual and professional work of specialists in the recreational field contributes to rescue and restructure society. Traditional games and singing games are then the tools used to facilitate relationships, contribute to the learning process, and exhibit skills. They are fundamental in a person's life since they are a social and cultural expression of how humans have adapted to their environment (Maestro, 2005). They do not take ethnicity, age, sex or social conditions into consideration. Traditional games are also a way of promoting health, improving motor, cognitive and emotional skills and a means of encouraging creativity and imagination and developing a sense of rhythm. Their goal is to attain a state of personal well-being. They are a way to release tension and accumulated energy and to get away from the daily routine. They represent a bridge to learn about oneself, the environment, values, habits, and traditions. In this document, readers will learn how traditional games are transmitted, what their characteristics are, why they are an important tool in today's society, how they are prepared, and how they can be revived and preserved.

KEY WORDS: traditional games, recreation, game, customs, traditions. 


\section{INTRODUCCIÓN}

Existen diversas etapas en la vida del ser humano, una de ellas es la infancia, en la cual el juego se convierte en la actividad de mayor trascendencia para el mundo que la rodea. Desde la cuna, cada persona empieza a descubrir de manera lúdica su propio cuerpo y sus posibilidades de movimiento. Se experimentan los sentidos trasformados en sensaciones y vivencias que empiezan a despertar los rasgos de personalidad. Conforme van creciendo su entorno se expande y por medio de la imitación se desarrollarán juegos como un fin en sí mismos. Es por tanto, que el juego desde la infancia hasta la adolescencia, desempeña un papel importante en el desarrollo de la persona, ya que, satisface sus necesidades de acción y expresión, se adquieren aptitudes físicas e intelectuales, aprende a relacionarse con los y las demás y con su entorno, así como valores y normas sociales que posteriormente serán necesarios en la vida adulta (Maestro, 2005).

En la sociedad tradicional, la pubertad significaba el final de la infancia, el abandono de la escuela y la integración hacia las responsabilidades de la vida y el trabajo adulto (Maestro, 2005). Actualmente este fenómeno no ha desaparecido del todo, aún los diferentes grupos etarios asumen el comportamiento que dicta las reglas sociales. El tiempo libre de los(as) jóvenes y las personas adultas se ve disminuido por el consumismo y la globalización, al igual que la tecnología, estos se apoderan de sus vidas. Desde el punto de vista social el juego ya no es tan importante en estas edades, es ahora un medio para desahogarse, mejorar la salud mental, contribuye a salir de la monotonía y estrés de la vida adulta.

Sin embargo, para las personas adultas mayores el juego es un rescate de recuerdos, de volver a vivir la infancia, las travesuras, la aventura y la alegría y un medio de compartir con la familia o el entorno, que muchas veces las han abandonado. Cabe señalar que no solo en las diferentes edades se presenta el juego. También a lo largo de la historia, los juegos han obtenido un papel fundamental y han estado presentes en todas las culturas y lugares. Son imprescindibles en algunas ocasiones para lograr cierto grado de desarrollo físico, psíquico y emocional. Igualmente, son un factor determinante en el proceso de socialización y aprendizaje del ser humano (Bantulá y Mora, 2002).

Por tanto, el juego es un instrumento que facilita y crea lazos interpersonales, sin importar raza, edad, sexo, condición social entre otros; una fuente generadora de salud; una ayuda para mejorar las destrezas motoras, cognitivas y emocionales; un medio para fomentar la creatividad, despertar la imaginación, desarrollar el sentido del ritmo; su objetivo es el bienestar personal; un estímulo para liberar tensiones y energía acumulada, así como para salir de la rutina diaria y un puente para el aprendizaje de si mismo, del entorno, de valores, costumbres y tradiciones.

Este trabajo tiene como propósito ofrecer una herramienta de antaño, los juegos tradicionales, que sirva como medio para la integración, interacción y rescate de la recreación en las aulas, las familias, los campos de juego y cualquier otro espacio que se utilice para la recreación.

\section{JUEGOS TRACIONALES}

Los juegos tradicionales son una excusa perfecta para aprender y relacionarse o como una prueba de demostración de habilidades. Forman parte inseparable de la vida de la persona y sobre todo, no es posible explicar la condición social del ser humano sin los juegos, ya que estos son una 
expresión social y cultural de la adaptación que ha protagonizado el ser humano en relación con su entorno (Maestro, 2005).

Reciben el nombre de juegos tradicionales todos aquellos conocidos por la gente de un determinado lugar, llevados a la práctica regularmente y que se conservan y transmiten de generación en generación, los cuales envuelven una gran cantidad de hechos históricos propios de ese lugar o zona de origen (Bustos y otros, 1999).

Este tipo de juegos populares traen consigo aspectos culturales de comportamiento y aprendizaje de valores, tareas cotidianas, normas y hábitos (Bustos y otros, 1999). Además, son actividades donde se permite rememorar eventos históricos de hace muchos años o siglos y conocer costumbres y tradiciones de los pueblos, que de generación en generación se han logrado recobrar y mantener... son un pasado que no se quiere olvidar (González, 2000).

\section{¿Cómo se Transmiten los Juegos Tradicionales?}

Según Maestro, (2005) la transmisión de los juegos tradicionales se ha dado de manera oral, generación tras generación. Y puede darse de dos formas: la vertical que se da a través de la familia y la horizontal por medio de la socialización. La familia es el primer lugar de aprendizaje. Por medio de sus diferentes miembros (abuelos, papás, abuelas, mamás o los hermanos y las hermanas) se introduce el entorno y se potencia el juego, a través de estímulos visuales, voces y sonidos. En la relación abuelo(a)-nieto(a) se crea un vínculo de protección, complicidad y de relación transgeneracional de gran importancia para la educación. Ellos y ellas aprenden con la experiencia de un referente vivo, a lo que se le puede llamar una transmisión generacional directa por imitación.

Conforme se va creciendo, las necesidades de acción y de relación producen un distanciamiento del núcleo familiar y el aprendizaje se continúa en la calle, en el patio de recreo, en la esquina del barrio. En ese momento, los juegos tradicionales cumplen su papel. Por una vía u otra los juegos como trompos, canicas, rayuela, yoyos, boleros, cronos, gallinita ciega, jackses, entre otros llegan hasta las personas y de esa manera se conservan en el tiempo (Maestro, 2005; Lavega y Olaso, 1999).

\section{Características de los juegos tradicionales}

Según González (2000) y Lavega y Olaso (1999) algunas características de estos juegos son:

Significan un medio de transmisión de valores y cultura.

Son de muy fácil comprensión, memorización y acatamiento. Sus reglas son flexibles.

No requieren de material costoso.

La gama es muy amplia y ofrecen muchas posibilidades para que todos y todas puedan participar.

Practicables en cualquier momento y lugar.

Representan un patrimonio cultural irremplazable.

Son un disfrute para la familia.

Son una fuente de motivación para grandes y pequeños.

Facilitan y estimulan el desarrollo de la sociabilidad entre generaciones, con nuestros pares y con el entorno más próximo.

Son la excusa para valorar y conocer otros juegos y tradiciones de otras culturas.

Favorecen la comunicación y adquisición del lenguaje. 


\section{¿Qué tipo de materiales se necesitan para jugarlos?}

Anteriormente, en los hogares se fabricaban los propios juguetes con palos, piedras, latas, cuerdas, hojas, mecates, entre otros. Es importante señalar que algunos de los juegos no precisan de un material específico y en caso de ser así, se puede reaprovechar cualquier objeto o material de los recursos existentes en el entorno inmediato (Bustos, 1999).

Los juegos tradicionales dan la posibilidad de que cada persona construya su juguete poniendo en práctica su ingenio y creatividad para utilizar el material disponible a su alcance. Convirtiendo al niño(a) en "artesano(a)" que de esa habilidad dependerá muchas veces su calidad como jugador (Maestro, 2005).

\section{Recobrando los Juegos Tradicionales}

A pesar de que en algunas sociedades los abuelos y abuelas pasan inadvertidos, Maestro (2005) destaca que ellos y ellas son un cúmulo de experiencias, un foco de atención, sabiduría y aprendizaje. A través de ellos (as) se pueden rescatar por medio de su memoria, los mayores juegos tradicionales, tarea que no solo es gratificante, sino da la posibilidad de utilizarlos como una herramienta para conseguir objetivos en proyectos específicos: antropológicos, históricos, deportivos, educativos, recreativos, entre otros.

El relato de los abuelos y las abuelas es el medio más importante para recolectar la información necesaria a fin de conocer cómo se jugaba, dónde, con qué y todo lo que involucre un juego específico. Ahora bien, es importante contrastar la información, jugarlo y conocer el contexto antropo-cultural, con el fin de valorar y conservar el patrimonio cultural a través del factor humano y social, vital para el individuo (Maestro, 2005).

A través de los relatos se pueden recobrar estos juegos, así como animar a grandes y pequeñitos a descubrir el valor que poseen y su historia. Además, la posibilidad de trasmitirlos y enriquecer la memoria de una región, una comunidad o una cultura e inclusive una igualdad de género, debido a que las diferencias generadas en la sociedad entre lo que el hombre y la mujer pueden practicar en el nivel lúdico es muy marcada aún en algunos lugares (Bustos y otros, 1999).

Dentro de los juegos tradicionales se puede encontrar una amplia gama de modalidades lúdicas: juegos de niños o niñas, canciones de cuna, juegos de adivinación, rimas, juegos de sorteo, juguetes y juegos de diversas variedades (Sisfontes, 1999).

\section{A continuación se presenta una serie de juegos tradicionales que se han podido recobrar:}

1. Ahorcado

2. Alto ("stop").

3. Alturita.

4. Aro.

5. Avispero.

6. Bolsillo.

7. Bolero.

8. Bolinchas o canicas y sus diferentes variantes como por ejemplo chócolas.

9. Bombetero. 
10. Burra lo largo y a lo alto.

11. Cachetadas con papel.

12. Cuartas.

13. Caballito de madera.

14. Cachirulos.

15. Carrera de aros.

16. Carrera de barquitos en el caño.

17. Carrera de cintas.

18. Carrera con limones.

19. Carrera de sacos.

20. Carrera de zancos.

21. Chapa con Manila (RUN RUN).

22. Cerbatanas.

23. Cromos.

24. Carros de madera.

25. Cola al burro.

26. Cuartel inglés.

27. Cuartas.

28. Cuerda o suiza.

29. Culebrita.

30. Elástico.

31. Enano, gigante.

32. Escondido y sus diferentes modificaciones.

33. Flecha o resortera.

34. Frío, caliente.

35. Fútbol con paleta y jugadores de clavo

36. Gallinita ciega.

37. Gato.

38. Guerra de frutas, boñiga, etc.

39. El Huevo y la cuchara.

40. Jackses.

41. Lanzar la herradura.

42. Los moros.

43. Luchas de naves (papel).

44. Mecate, sencillo, doble, triple.

45. Países.

46. Palo ensebado.

47. Papalote.

48. Papa caliente.

49. Pique tres.

50. Policías y ladrones.

51. Póngale la cola al burro.

52. Puritos.

53. Quedó y todas sus variantes.

54. Sortijita.

55. Raya.

56. Rayuela, de diferentes formas, colores.

57. Resbalar con cartón.

58. Tablero con chapas. 
59. Teléfono chocho.

60. Trecillo.

61. Tres chapas.

62. Trompos: zapita (achatado y pequeño), macho (alargado y grande) y sus diferentes variantes trucos con el trompo, guerritas entre otros.

63. Un, dos, tres queso.

64. Yoyo, con todos sus trucos.

(Hernández, 1987; González, 2000; Sisfontes, 1999; Lavega y otros, 1999; Ramírez, 1993).

\section{Y algunas rondas tradicionales son:}

1. Ambo matarilerileron.

2. Arroz con leche.

3. El gato y el ratón.

4. Juguemos en el bosque.

5. El reloj (los esqueletos).

6. Guayabita del Perú.

7. Hueso vamos a comer.

8. La casa de Don Juan.

9. La mar.

10. La muerte.

11. La pájara pinta.

12. La semana.

13. Las tinajitas.

14. Lero lero.

15. Llego carta.

16. Los pollos de mi cazuela.

17. Mirón mirón.

18. Oba.

19. Osito osito.

20. Pájara pinta.

21. Pajarito pito pito

22. Paris o nonis

23. Pisi Pisi araña.

24. Piedra, papel o tijera

25. Rabito conejo.

26. Ronda de amistad.

27. Ron maca ron.

28. Tinajas.

29. Tin marín.

30. Zapatito cochinito.

(Hernández, 1987; González, 2000; Sisfontes, 1999; Lavega y otros, 1999; Ramírez, 1993). 


\section{CONCLUSIONES}

Los juegos y rondas tradicionales son una forma de rescatar: la memoria de una región, el testimonio de una comunidad, las costumbres de una tierra y las tradiciones que de generación en generación formarán una historia.

Motivar la recreación familiar a través de los juegos tradicionales no solo favorece la interacción de las y los miembros, sino que también amplía la gama de actividades en las cuales se pueden conocer, divertir, reír, intercambiar ideas y compartir sanamente.

Por medio de la utilización de los juegos y rondas tradicionales se puede fomentar la creatividad, mejorar la salud (física, mental y emocional), fortalecer los valores y actitudes, incrementar las destrezas y habilidades de todas las poblaciones sin distinción de raza, credo, edad, discapacidad o identidad sexual.

El juego continúa desempeñando un papel muy importante en el desarrollo integral de los infantes, un medio de formación para las personas adolescentes, un factor de unión entre las personas jóvenes y adultas y un conocimiento para enseñar, en las personas adultas mayores.

Los juegos y las rondas tradicionales son otra herramienta más de la gama de actividades recreativas que pueden contribuir para transmitir, rescatar y mantener los valores culturales de generación en generación.

En el ámbito educativo, los juegos y las rondas tradicionales se convierten en una herramienta de fácil acceso, de bajo costo y con una diversidad tal, que puede ser utilizado en cualquier ocasión y/o lugar y por cualquier persona.

\section{REFERENCIAS}

Bantulá, J y Mora, J. (2002). Juegos multiculturales: 225 juegos tradicionales para un mundo global. Barcelona: PAIDOTRIBO.

Bustos, M., Carrión, M., García, J., Guzmán, J., Irigoyen, A., Larraya, I., López, J., Maestro, F., Martínez, F., Maseda, J., Muriel, J., Ruiz, M., Sánchez, J., Valle, R., Velásquez, C. y Zoroza, A. (1999). Juegos: una propuesta práctica populares para la escuela. España: Pila Teleña.

González, A. (2000). Tin Marín ja jugar! San José, Costa Rica: EUNED.

Hernández, M. (1987). Juegos tradicionales costarricenses. Costa Rica: Talleres gráficos de Offiprint Industrial S.A.

Lavega, P.y Olaso, S. (1999). 1000 Juegos y Deportes populares y tradicionales: tradición jugada. Barcelona: PAIDOTRIBO.

Maestro, F. (2005). Juegos tradicionales. España.

Sisfontes, P. (1999). Juegos y Rondas. Documento en la Biblioteca Clemencia Conejo. Escuela Ciencias del Deporte, Universidad Nacional.

Ramírez, O. (1993). Al rescate de las rondas y los juegos tradicionales. Costa Rica: Impresos Costa Rica 2000 
Fecha de recepción del artículo: 11 de octubre del 2007.

Fecha de aceptación del artículo: 13 de marzo del 2008.

Fecha de publicación del artículo: 31 de julio del 2008.

\section{cc) (†)}

Artículo de la Revista MHSalud de la Universidad Nacional, Costa Rica protegido por Licencia Creative Commons Attibution-NonComercial-NoDerivs 3.0 Costa Rica,

Para más información visite www.una.ac.cr/MHSalud

Permissions beyond the scope of this license may be available at revistamhsalud@una.cr 